\title{
Superregeneration Revisited: From Principles to Current Applications
}

\author{
Pere Palà-Schönwälder, Member, IEEE, Jordi Bonet-Dalmau, Member, IEEE, Francisco del Águila-López, \\ F. Xavier Moncunill-Geniz and Rosa Giralt-Mas
}

\section{INTRODUCTION}

Wireless communications play a central role in our modern connected lives; at the same time, they constitute a very broad and deep area of research. The elements that make wireless communications possible are a transmitter, which is able to place information on electromagnetic waves; a medium able to transport these waves; and, finally, a receiver which extracts the information from the-usually very small-amount of energy it is able to collect from the medium.

As there are a myriad of communications taking place simultaneously, a communications receiver has the additional task of isolating the desired signal from others. Hence, a receiver is a device that 1 ) is able to reject the unwanted signals as much as possible, 2) amplifies the received signal until it is suitable for further processing, and 3) reconstructs the original information that was sent by the transmitter as faithfully as possible. In a nutshell, a receiver provides selectivity, gain, and demodulation capabilities.

Deciding whether a small received signal represents a digital one or a digital zero is not a trivial task. To illustrate how this works in a real-life context, consider the task of finding out if a fluorescent tube light, which is hanging from the ceiling, is deviated to the right or to the left of the exact center of a long table (Fig. 1). Of course, if the deviation is big enough, this is easy but how can this be done for very slight deviations? One way to proceed is to use a plumb bob with the tip hanging near the table surface and optical magnification to decide whether the tip of the bob lies to the right or to the left of the table's center line. Strong (and expensive) magnification will be needed if the light's deviation is extremely small.

An alternative is to use a rod with two sharp tips and build an inverted pendulum, letting the rod rotate around its bottom tip. Place the bottom tip on the center line, place the upper tip at the fluorescent tube and let the system evolve! If you take a photograph of the rod at some fixed instant after it has been released, its inclination (for small values) will be proportional to its initial inclination. Furthermore, if you just wanted to know if the starting inclination was positive or negative, just let the rod hit the table-you will not have to wait long!

Manuscript received xx, 2018; revised xx, 2018. Work supported by Spanish Grant TEC2015-65748-R (MINECO/FEDER).

The authors are with the Department of Mining, Industrial and ICT Engineering (EMIT), Manresa School of Engineering (EPSEM), Universitat Politècnica de Catalunya (UPC), 08242 Manresa, Spain (e-mail: pere.pala@upc.edu).

Copyright (c) 2018 IEEE. Personal use of this material is permitted However, permission to use this material for any other purposes must be obtained from the IEEE by sending an email to pubs-permissions@ieee.org.

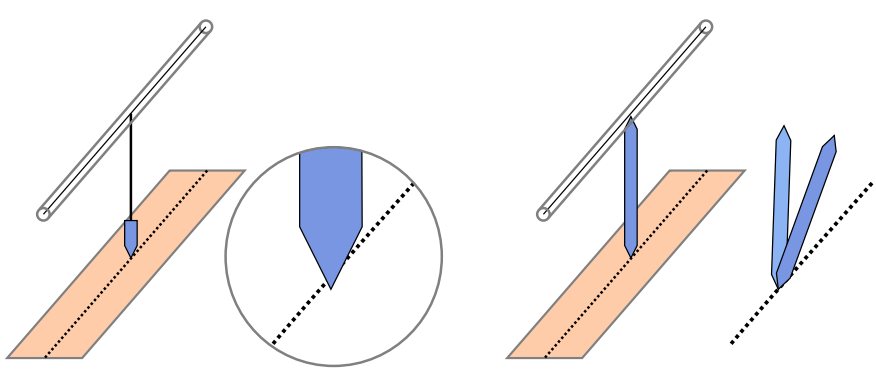

Fig. 1. The task of determinig wether a fluorescent tube light is slightly displaced to the left or to the right of the exact center of a table can be done in two ways: by optical magnification or exploiting instability.

Classical receivers may be seen as following the optical magnification method: a chain of amplifiers is eventually able to provide the $120 \mathrm{~dB}$ of gain required to bring the input $\mu \mathrm{V}$ level signals up to $\mathrm{V}$ levels. However, several gain stages (at different frequencies) are required for this, with their inherent component cost, reliability, size, and power consumption. Conversely, superregenerative (SR) receivers exploit instability, as in the previous example. Just by letting time pass, an initially small signal can be arbitrarily amplified with just one active stage.

SR receivers were introduced a century ago [1], and were widely used during World War II in pulse transponders for radar identification of ships and aircraft. Despite having a structurally simple architecture and the basis being covered in depth several decades ago [2], the matter is subtle and complex enough that it has never been understood by more than a handful of people at a given time [3]. This paper tries to shed some light on the principles of SR receivers, concentrating on the qualitative aspects. In what follows we have tried our best to present the most significant pieces of information, providing a framework into which more advanced details can be fitted. The reader is encouraged to first get an overall view of the subject by skipping those elements that may initially seem too obscure. The very interested reader can find further support material in the references.

The previous rod example had real poles, with a dominant first-order response given by a single real unstable pole. A pair of complex conjugate unstable poles is required to achieve the bandpass characteristics of standard communication receivers. In what follows, we will start by concentrating on some results for first-order systems, which can be seen as a lowpass equivalent of the second-order bandpass case. 


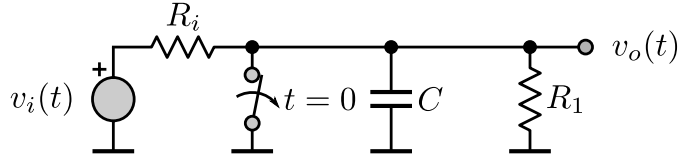

Fig. 2. This first-order system becomes unstable when $-R_{i}<R_{1}<0$.

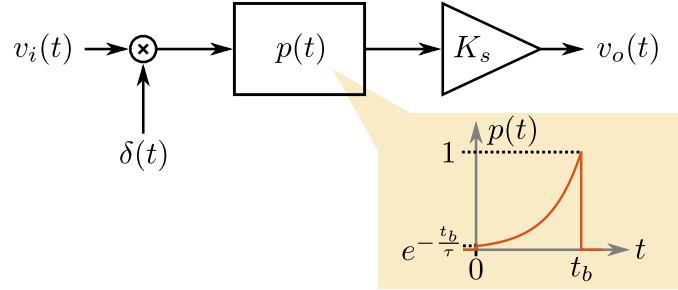

Fig. 3. Equivalent block diagram of a first-order unstable system for slowly varying inputs. The input signal is sampled, shaped by $p(t)$ and amplified by a factor $K_{s}$

\section{FIRST-ORDER INSTABILITY}

Even if it were not the first result in chronological terms, the analysis of an unstable first-order response is interesting because it provides some light for further discussion of the SR principle. We will use this to present some key ideas that will be extended later.

A circuit that displays a behavior similar to the rod example is given in Fig. 2 provided that the overall resistance $R=$ $R_{1} \| R_{i}$ (or the conductance $G=1 / R$ ) is negative, i.e., if $-R_{i}<R_{1}<0$. When the switch is opened, the response of the circuit to a sinusoidal input $v_{i}(t)=V \cos (\omega t+\alpha)$ (which includes the particular case $\omega=0$ and $\alpha=0$, i.e., pure dc) is the sum of a natural and a forced term, i.e.,

$$
v_{o}(t)=v_{n}(t)+v_{f}(t)
$$

given by [4]

$$
\begin{gathered}
v_{n}(t)=V|H(\omega)| \cos (\alpha+\varphi) \exp \left(\frac{t}{\tau}\right) u(t) \\
v_{f}(t)=V|H(\omega)| \cos (\omega t+\alpha+\varphi) u(t),
\end{gathered}
$$

with $\tau=-R C$ (which is a positive value). As $t$ increases, the natural response soon becomes the dominant term of the response. If the system is left in the unstable state until $t=$ $t_{b}$, the output signal is an exponentially growing pulse that represents the input signal $v_{i}(t)$ at $t=0$ (after being filtered by $H(\omega)$ ), amplified by a factor

$$
K_{s}=\exp \left(\frac{t_{b}}{\tau}\right)
$$

which is responsible for the huge signal gain. The frequency response term $|H(\omega)|$ (and $\varphi=\arg H(\omega)$ ) is given by a first-order function with a right half plane pole at $s=1 / \tau$ [4].

From a signal processing point of view, if we ignore the forced response and consider slowly varying inputs $(\omega \ll 1 / \tau)$ the system is equivalent to the diagram in Fig. 3, where the block labeled $p(t)$ describes a linear, time-invariant system with impulse response $p(t)$.

If the switch is closed at $t=t_{b}$ and reopened at a new sampling instant $t=T_{s}$ the preceding analysis is valid with

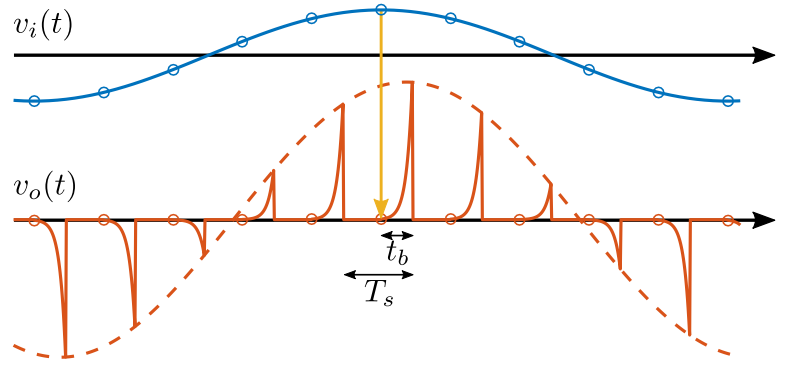

Fig. 4. Waveforms $v_{i}(t)$ and $v_{o}(t)$. Each input sample produces an exponentially rising pulse.

the new time origin. Hence, if the switch is opened and closed periodically, the response will be a succession of exponentially growing pulses whose peak amplitude is proportional to the input signal sampled at the instants when the switch is opened, as shown in Fig. 4.

\section{A. Applications}

This principle, with the negative resistance implemented by a humble TL081 Operational Amplifier, which has a GB of $4 \mathrm{MHz}$, has been used to build baseband amplifiers in the $\mathrm{kHz}$ range. With the same active element, a $455 \mathrm{kHz}$ downconverting mixer with $46 \mathrm{~dB}$ conversion gain has been built [4]. The same principle has been recently used to build an electrocardiogram amplifier [5] and UWB detectors [6], [8] in the $\mathrm{GHz}$ range. On the other hand, the well-known flip-flop (FF) circuit is based on the same principle, but its output is usually observed in one of its saturated states. FFs are designed to quickly resolve into a logical zero or one, with metastability being the only significant analog issue.

\section{B. Gain, Bandwidth, and Power Consumption}

The resulting gain depends on the time allowed for the response to grow, $t_{b}$, which is limited by the maximum period to comply with Nyquist's sampling criterion. Given the signal bandwidth $B$, the sampling frequency $k B$, and considering that $t_{b}$ is a fraction $\delta$ of the sampling period, we get the result [4]

$$
G(\mathrm{~dB}) B \approx 8.7 \delta /(k \tau)
$$

Note that, in contrast to standard amplifier stages, it is not the gain-bandwidth product $G B$ that is almost constant for a given amplifier, but $G(\mathrm{~dB}) B$. Hence, the $G B$ product of a SR amplifying stage can exceed the $G B$ product of the active element. The ability to achieve high gain is especially attractive at higher frequencies, where devices have limited gain. To achieve a specified gain, a cascade of $N$ stages is often required, requiring $N$ times more power consumption and cost. In contrast, a single SR gain stage can provide the same overall gain.

The gain in $\mathrm{dB}$ is linearly proportional to the time spent in the unstable state. A single active element can replace a chain of low-gain stages. 

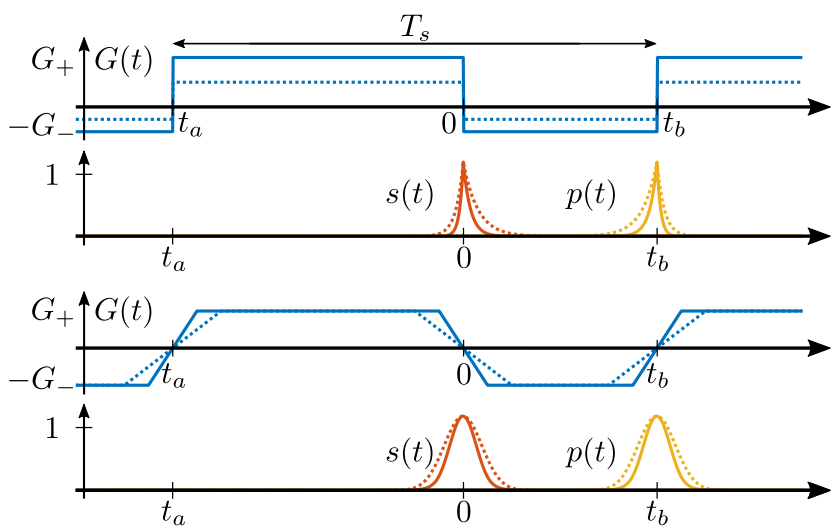

Fig. 5. Variable conductance, sensitivity function, and generated pulses in the step-controlled (top) and the slope-controlled (bottom) states.

\section{Gradual Conductance Variation}

In this first example, we have seen that the conductance changes abruptly from infinity to a negative value due to the switch. A natural question is what happens when there is a gradual change between finite values. This has been addressed in [6], [8] in the UWB context for input pulses given by $v_{i}(t)=V p_{i}(t)$. The output in this case is a different pulse,

$$
v_{o}(t)=V K_{r} K_{s} p(t),
$$

where we have two gain terms $K_{r}$ and $K_{s}$ and a normalized pulse shape $p(t)$. The regenerative gain $K_{r}$ is given by [8]

$$
K_{r}=\frac{1}{R_{i} C} \int_{t_{a}}^{t_{b}} p_{i}(\tau) s(\tau) d \tau,
$$

where the sensitivity function

$$
s(t)=\exp \left(\frac{1}{C} \int_{0}^{t} G(\tau) d \tau\right)
$$

has a determining role in the response. For instance the SR gain is $K_{s}=1 / s\left(t_{b}\right)$ and it can be shown that $p(t)=$ $s\left(t_{b}\right) / s(t)$. Furthermore, $s(t)$ has its maximum at $t=0$, the instant where the conductance crosses zero. This means that the system is only sensitive to the input during a window around $t=0$ (Fig. 5).

When the conductance changes abruptly between infinity and $-G_{-}$, as happened with the switch in Fig. 2, the sensitivity function is a right-handed exponential. When the conductance changes abruptly between $G_{+}$and $-G_{-}$, which is called the step-controlled state in the literature [2], we get two exponential arms (Fig. 5, top), which are symmetric when $G_{+}=G_{-}$. When the conductance changes linearly between $G_{+}$and $-G_{-}$, which is known as the slope-controlled state, we get a Gaussian sensitivity function. In the usual case when $G(t)$ has even symmetry around $t_{b} / 2$ the pulses generated by the SR system exhibit a similar shape, given by $p(t)=s\left(t_{b}-t\right)$ (Fig. 5).

Also note that these results are obtained for baseband input pulses, meaning that there is no sinusoidal variation in the input. A sinusoidal variation could be included in $p_{i}(t)$ and we would get the same results as in the first part of this section, with a frequency-dependent term $H(\omega)$ arising from $K_{r}$ that could be brought out explicitly to account for the frequency

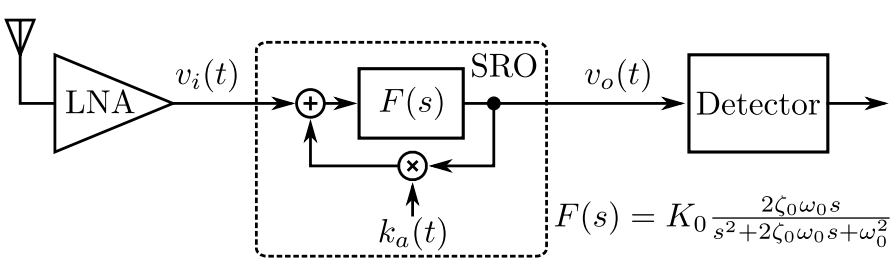

Fig. 6. Block diagram of an SR receiver.

response of the overall system, as has been done in (2) and will be done later.

Summarizing, the main conclusions thus fare are:

- The relative output amplitudes for different input pulses are only determined by $K_{r}$, which is the correlation between the input pulse and $s(t)$ evaluated during the observation frame given by $t=0$. The sensitivity function makes the system sensitive to the input only at certain instants or, more precisely, time windows, during what is often called the sensitivity period. Hence, one often talks about the sampling action of SR systems. Signals outside of this windows have little effect on the response.

- The shape of $G(t)$ has a strong influence on the sensitivity function, which, in turn, determines which portions of the signal are observed.

- Gain is mostly achieved through instability, by the SR gain factor $K_{s}$.

\section{THE SUPERREGENERATIVE OSCILlator: SECOND-ORDER INSTABILITY}

A conventional SR receiver is a periodically unstable second-order system. The basics of its operation have been covered by Armstrong in 1922 [1] and, some decades later, in more comprehensive form, in Whitehead's book in 1950 [2]. In these and most other references, results are presented for particular circuits, in a way similar to what has been done in Section II. A more abstract and general viewpoint, suitable for several oscillator topologies and providing a framework for advanced applications, can be found in [10], which will be used in the following discussion. Note that the first-order case can also be seen in this way.

A SR receiver can be shown to be equivalent to the block diagram given in Fig. 6. The core of the receiver is the superregenerative oscillator (SRO), where the gain in the feedback loop, $k_{a}(t)$, is controlled by a signal (called a quench signal) so that when the instantaneous damping factor of the closed loop, $\zeta(t)$, is negative (or positive), the output is an exponentially growing (or decaying) sinusoid. The constant quench signal that makes the closed loop marginally stable (Barkhausen criterion) is the one that makes $\zeta=0$. The lownoise amplifier (LNA) is transparent from the signal processing point of view and serves the usual low-noise objective in a communications receiver and avoids oscillator reradiation. The detector block may be different depending on the targeted modulations and will be discussed later. This block diagram could also represent the first-order case, replacing the filter with a first-order low-pass one. 
TABLE I

STEP-CONTROLLED AND SLOPE-CONTROLLED STATES

\begin{tabular}{|c|c|}
\hline Step-Controlled & Slope-Controlled \\
\hline$\zeta(t)= \begin{cases}\zeta_{+} & \text {if } t<0 \\
-\zeta_{-} & \text {if } t>0\end{cases}$ & $\begin{array}{l}\zeta(t) \text { is irrelevant. } \\
\text { Only } \beta=-\dot{\zeta}(0) \text { matters. }\end{array}$ \\
\hline$s(t)= \begin{cases}\exp \left(\omega_{0} \zeta_{+} t\right), & t<0 \\
\exp \left(-\omega_{0} \zeta_{-} t\right), & t \geq 0\end{cases}$ & $s(t)=\exp \left(\frac{-\omega_{0} \beta t^{2}}{2}\right)$ \\
\hline
\end{tabular}

The SRO is described by a differential equation which, for usual conditions, may be approximated as

$$
\ddot{v}_{o}(t)+2 \zeta(t) \omega_{0} \dot{v}_{o}(t)+\omega_{0}^{2} v_{o}(t)=K_{0} 2 \zeta_{0} \omega_{0} \dot{v}_{i}(t),
$$

with $\zeta(t)$ given by

$$
\zeta(t)=\zeta_{0}\left(1-K_{0} k_{a}(t)\right) .
$$

For a parallel resonant circuit, $\zeta$ is proportional to the conductance, i.e., $\zeta(t)=G(t) /\left(2 C \omega_{0}\right)$ [10]. So, the qualitative description in Fig. 5 is valid, changing $\zeta(t)$ by $G(t)$. Under reasonable assumptions, the response of this system to an input pulse applied in the interval $t_{a} \leq t \leq t_{b}$, given by

$$
v_{i}(t)=V p_{i}(t) \cos (\omega t+\phi),
$$

is [10]

$$
v_{o}(t)=V K|H(\omega)| p(t) \cos \left(\omega_{0} t+\phi+\angle H(\omega)\right),
$$

which is similar to (6), with $K=K_{0} K_{r} K_{s}$. The constant $K_{0}$ is the so-called feedforward gain and deserves no further comments. The term $K_{r}|H(\omega)|$ (showing explicit dependence with $\omega$ ) is an extension of (7) to the bandpass case, with the sensitivity curve $s(t)$ now given by

$$
s(t)=\exp \left(\omega_{0} \int_{0}^{t} \zeta(\tau) d \tau\right) .
$$

The normalized frequency response term $H(\omega)$ is now bandpass, centered at $\omega_{0}$, and will be discussed later.

The manner in which the system evolves from the stable to the unstable state determines the sensitivity function, which, in turn, determines almost all the significant parameters of the SRO.

In the second-order case, $s(t)$ also has a determining role on several parameters of the SRO. Table I shows $s(t)$ corresponding to the step-controlled and slope-controlled states. As was qualitatively shown in Fig. 5, in the step-controlled state $s(t)$ has exponential arms and in the slope-controlled state $s(t)$ becomes a Gaussian. A particular case of the slope-controlled state is the sawtooth waveform, which has a constant-slope damping factor $\zeta(t)=-\beta t$.

As a final remark, note that the results in (12) are based on assumptions which hold in most applications. More precise expressions, suitable for very high quench rates, have been derived in [9] and [11].

\section{A. Amplitude Detection}

For many decades, the most usual way to operate an SR receiver was to perform envelope detection of the RF pulses followed by a low-pass filter (Fig. 7). This is still done in the cheapest remote-control devices. This technique allows the detection of amplitude modulated signals, either analog or digital.

1) Advantages: Overall simplicity. No synchronization is needed at the receiver level: this is done later, during the signal processing chain.

2) Disadvantages: Reception bandwidth is much bigger than signal bandwidth. Typically, 5 to 10 pulses are needed per received bit, which means that the receiver's bandwidth is 5 to 10 times higher than with synchronous detection, which will be discussed later.

\section{B. Frequency Detection}

The classical way to detect frequency modulations was to perform an FM to AM conversion thanks to significant variations of $|H(\omega)|$ for the range of instantaneous frequencies to be detected [12]. The higher the index of modulation, the greater the amplitude differences that are obtained. A technique that has been used to detect FM-UWB is based on two SROs, each tuned to one of the frequencies to be detected [13], and choose which one gives greatest amplitude. This is only feasible if both frequencies are sufficiently separated, as in an UWB context. If they are close, the interaction between oscillators makes this approach unfeasible. As an alternative, a single SRO can be quenched at twice the frequency, tuned to $f_{1}$ during the even quench periods and to $f_{2}$ in the odd ones [14]. The output giving higher amplitude tells the transmitted frequency. However, this means doubling the quench frequency and the equivalent noise bandwidth. An alternative to detect narrowband FM will be presented in section VIII.

\section{Synchronous Amplitude Detection}

If the quench signal can be synchronized with the signal to be detected, a single quench cycle is enough to detect each received bit. This approach was shown in [15], demonstrating high-speed operation of the SR principle, and has been used extensively afterwards [7], [16], [17]. With this approach, the bandwidth penalty of typical SR receivers is reduced by the oversampling factor of 5 to 10 . There is, however, still a loss because the input signal outside of the observation window is lost [15], [18]. In fact, the transmitted power can usually be reduced by $10 \mathrm{~dB}$ by pulsing the transmitter with a $10 \%$ duty cycle with no effects on the receiver. Furthermore, if the sensitivity function is Gaussian, the SR receiver can be a matched filter for a transmitted signal consisting of amplitudemodulated Gaussian pulses [18]-[20] whose width is typically $10 \%-20 \%$ of the bit length.

With suitable quench techniques it is possible to shape $\zeta(t)$ adequately, making $s(t)$ wider in the time domain. This means that less energy is lost, or, from a frequency response point of view, that the equivalent noise bandwidth is smaller. Some ideas on this will be discussed in section IV. 
The sensitivity function explained

The sensitivity function can be obtained analytically, but there is also an intuitive view. The linear, time-varying SR system can be characterized by its impulse response $h(t, \tau)$. Let's consider the first-order case with rectangular quench, i.e., the step-controlled state. An impulse at $t=0$ gives an exponentially growing response (yellow trace). An impulse happening later (violet traces) will give the same kind of response, but its effect at $t_{b}$ will be much less. An impulse before $t=0$ will be first exponentially attenuated until $t=0$ and its effect at $t_{b}$ will also be less than when it was placed at $t=0$.

The sensitivity function describes the relative contribution of each time instant to the overall response. So, $s(t)$ is the normalized response to an impulse placed at $\tau=t$ evaluated at $t=t_{b}$. Or, in terms of $h(t, \tau), s(t)=h\left(t_{b}, t\right) / h\left(t_{b}, 0\right)$.
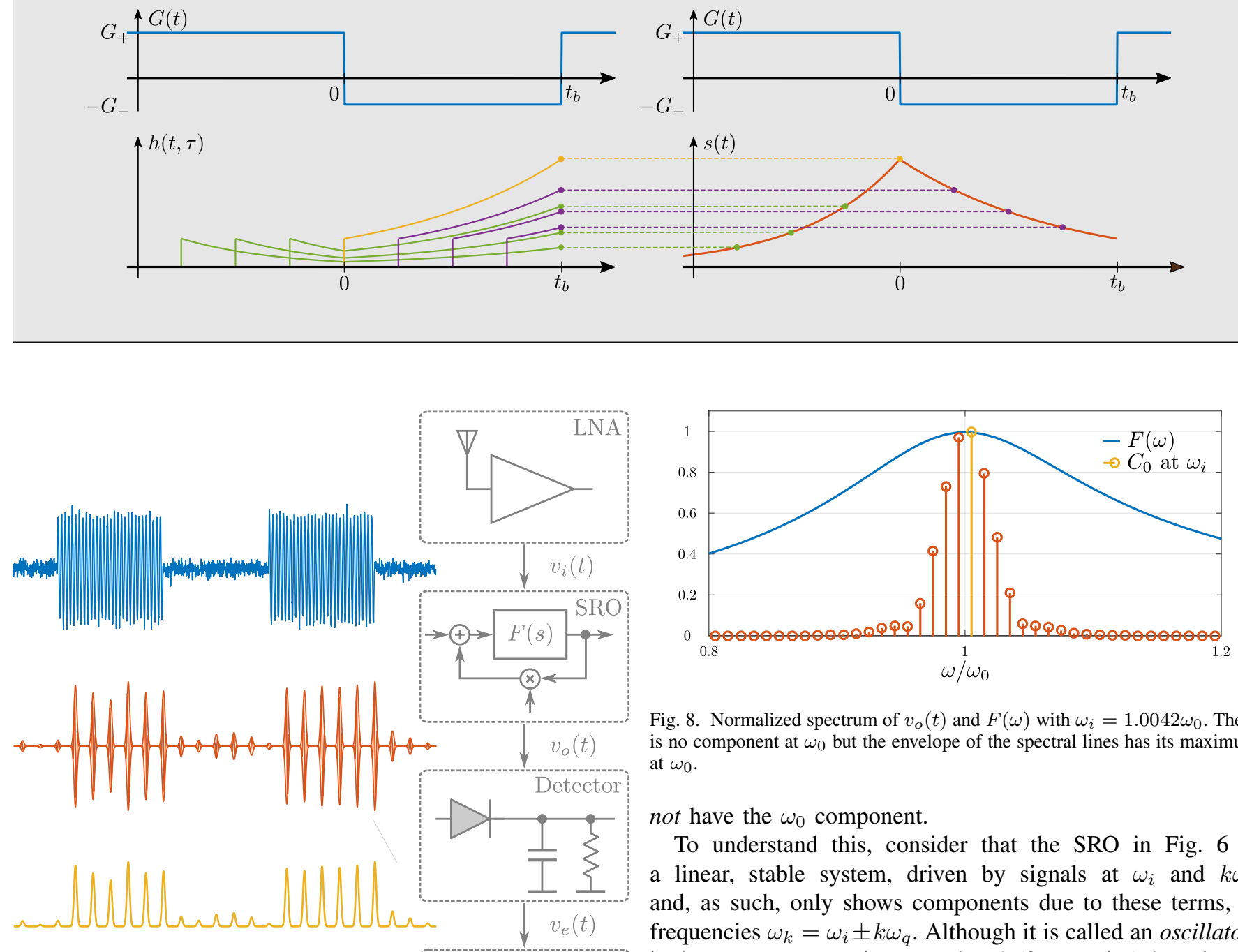

Fig. 8. Normalized spectrum of $v_{o}(t)$ and $F(\omega)$ with $\omega_{i}=1.0042 \omega_{0}$. There is no component at $\omega_{0}$ but the envelope of the spectral lines has its maximum at $\omega_{0}$.

not have the $\omega_{0}$ component.

To understand this, consider that the SRO in Fig. 6 is a linear, stable system, driven by signals at $\omega_{i}$ and $k \omega_{q}$ and, as such, only shows components due to these terms, at frequencies $\omega_{k}=\omega_{i} \pm k \omega_{q}$. Although it is called an oscillator, it does not generate its own signal (from noise) but simply processes its input signals. The only significant feature that happens at frequency $\omega_{0}$ is the maximum of the envelope of the output spectrum. Figure 8 shows a typical spectrum, with components $C_{k}$ at frequencies $\omega_{k}=\omega_{i}+k \omega_{q}$ following an envelope whose maximum is at $\omega_{0}$ [21].

The SRO also processes noise, which can be modeled as

Fig. 7. Classic amplitude detection process: an envelope detector followed by a low-pass filter (LPF).

An SRO with a Gaussian sensitivity function can be a matched filter for Gaussian pulses.

\section{Spectrum of the SRO Output Pulses}

Even if the response (12) of the SRO has a $\cos \left(\omega_{0} t\right)$ term, in sinusoidal steady state, the response to a $\cos \left(\omega_{i} t\right)$ input does a continuous distribution of spectral lines with random phase, with each differential input line producing a similar response, giving a continuous (as corresponds to a random signal) bellshaped curve. In practice, we have a superposition of signal plus noise and a spectrum analyzer shows images such as in Fig. 9.

Some effects of the changes in the parameters of the quench signal can be easily seen on a spectrum analyzer. For instance, if the quench signal has insufficient positive area, the SRO pulses are not quenched sufficiently before a new cycle starts 

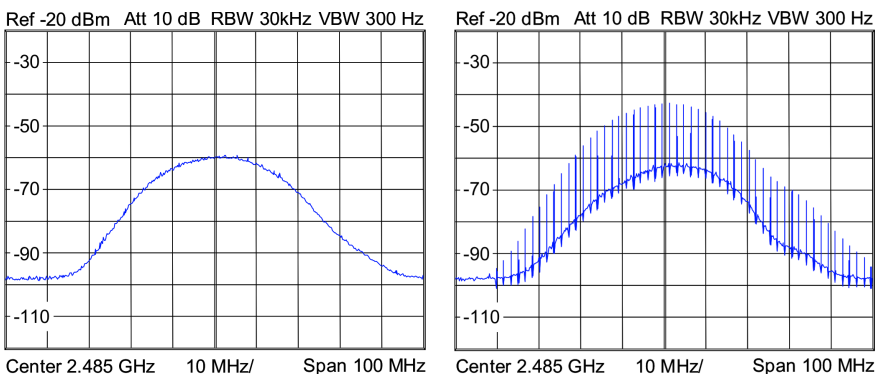

Fig. 9. Measured spectrum of a $2.4 \mathrm{GHz}$ SRO due only to noise (left) and to a $\mathrm{CW}$ signal [22].

and each pulse has an effect on the next one. When the remnants of the previous cycle are not negligible, the SRO is said to operate with appreciable hangover. This is difficult to assess in the time domain, but the superposition of attenuated and delayed versions of the same information introduces a ripple in the spectrum, a feature that is easily spotted on the spectrum analyzer. When hangover increases further, each new pulse is completely determined by the previous one. This becomes visible with spectral lines appearing in the absence of input and the SRO is said to be operating in coherent state. In this case, the mean $\zeta$ is negative.

\section{QUENCH TECHNIQUES}

The instantaneous variation of the damping factor $\zeta(t)$ is the most important parameter in SRO operation. Control of $\zeta(t)$ is typically achieved by varying the gain of an amplifier acting on its bias circuit. This has an indirect effect on $\zeta(t)$, but high gain means instability, i.e., negative $\zeta$ and low gain positive $\zeta$. The relation between control voltage and $\zeta(t)$ is often non-linear. Also, given the exponential nature of the expressions, in practice it is not easy to obtain the exact behavior predicted by theoretical results. Tolerances and, most important, temperature effects tend to destabilize the resulting waveforms. For instance, to keep the SR operating in linear mode some kind of feedback is necessary. But, keeping this often overlooked facts in mind, it is still possible to adjust the quench waveform to achieve certain desired properties.

Briefly [10], a) the slope of $\zeta$ at the zero crossing determines the receiver's bandwidth, b) the negative area determines gain, and c) the positive area provides attenuation of the previously generated pulse. If the attenuation is not enough, there is hangover, which means that the response of the current quench cycle will contribute to the output in the next cycle. Moreover the overall area has to be positive for global stability.

A simple-to-generate and frequently used quench signal is a sinusoid with adjustable amplitude and offset. Adjusting amplitude changes the slope of the zero crossing; hence it influences the bandwidth. Higher amplitude means higher bandwidth, but also higher gain (due to the increased negative area). Adjusting the offset also changes slope and area and has a consequence on bandwidth and gain. Hence, both adjustments are not orthogonal.

Other waveforms such as sawtooth, exponential, and others have been described and tested [2]. A sawtooth signal has the desirable property of having constant (and relatively low) slope

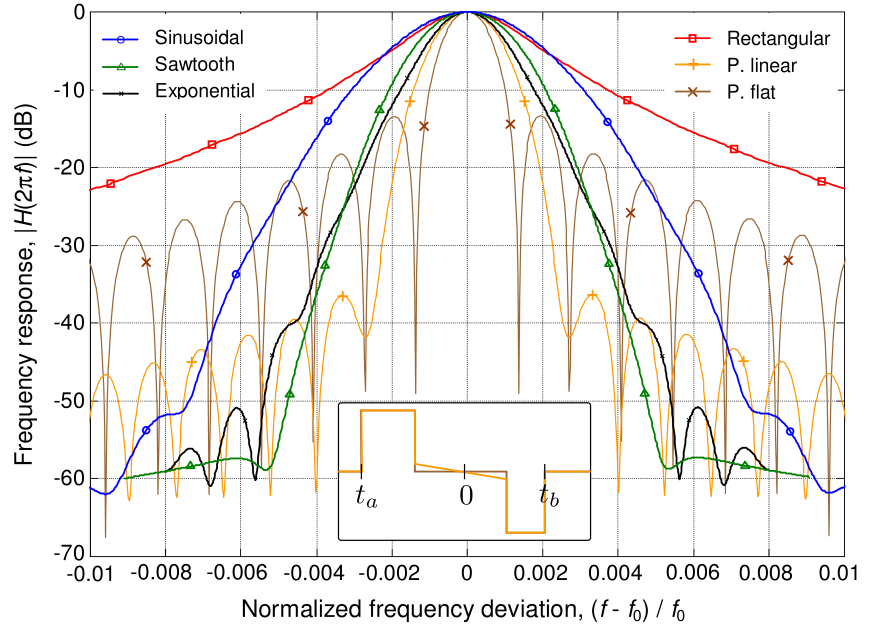

Fig. 10. Frequency responses for different quench signal types. Conditions: feedback filter $\mathrm{Q}=50, f_{q} / f_{0}=10^{-3}, K_{r} K_{s}=60 \mathrm{~dB}$ and $\overline{\zeta(t)}=0.002$.

regardless of offset, which makes it attractive. What could be interpreted as an optimum quench waveform, presented in [2] and revisited in [23] (see the inset in Fig. 10), is a piecewise linear shape with a slow zero crossing (to enhance selectivity), a strong negative peak (to achieve high gain), and a strong positive peak (to effectively quench the oscillator).

Figure 10 shows the simulated resulting frequency responses for several quench waveforms: sinusoidal, sawtooth, exponential, rectangular, optimum piecewise linear, and the idealized limiting case of the optimum piecewise linear with zero slope during as high a fraction of the quench period as possible. There are several considerations and details around this figure that this paper will not delve into. Still, this figure is expected to show the variety of responses that can be achieved. In any case, to get meaningful results, several conditions have to be specified: in the case of Fig. 10, the reception frequency was 1000 times the quench frequency, the gain $K_{r} K_{s}$ was kept constant at $60 \mathrm{~dB}$ and the mean damping (which determines hangover) was kept at 0.002 .

For a sawtooth quench signal, the frequency response (down to $\sim-K_{r} K_{s} \mathrm{~dB}$ ) is given by

$$
H(\omega)=\frac{\omega}{\omega_{0}} \exp \left(-\frac{\left(\omega-\omega_{0}\right)^{2}}{2 \omega_{0} \beta}\right) .
$$

To get an insight on the trade-offs that occur when selecting the quench signal, Fig. 11 shows the $3 \mathrm{~dB}$ bandwidth for several quench shapes as a function of the quench frequency, keeping the gain $K_{r} K_{s}$ constant. In principle, lower quench frequency means lower slope $\beta$ (which means lower bandwidth), but also higher negative area (which means higher gain). Hence, to keep a constant gain, amplitude has to be decreased, which again means lower bandwidth.

Furthermore, the operation limits in Fig. 11, where each waveform is shown for limited ranges, are worth some comments. On the one hand, the quench frequency can not exceed an upper limit because of hangover, which increases at higher quench frequencies. To keep hangover constant, higher losses have to be introduced but this has a limit. In the scheme of Fig. 6 very high losses means that the amplifier has to 


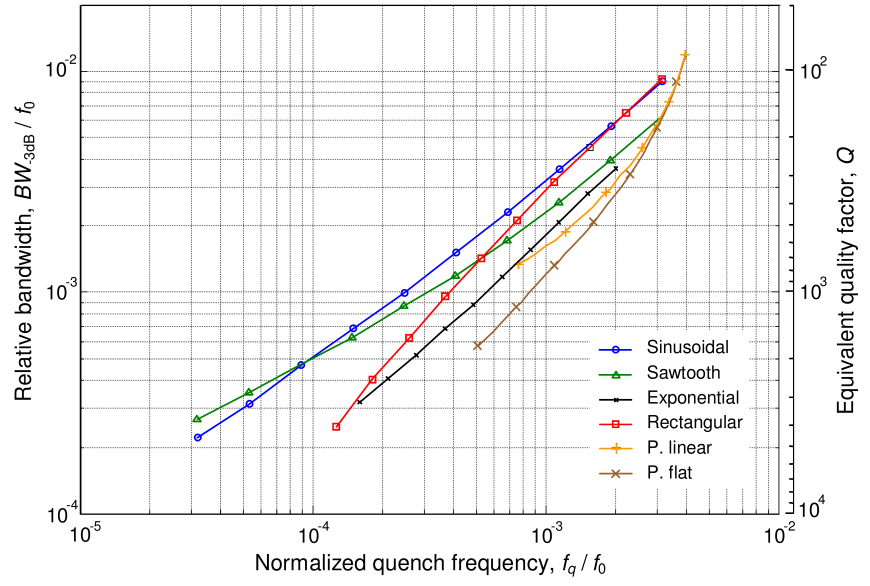

Fig. 11. $3 \mathrm{~dB}$ bandwidth (and equivalent $Q$ ) for different quench signal types at constant gain. Note that the range of available quench frequencies depends on the quench shape. Conditions: feedback filter $\mathrm{Q}=50, K_{r} K_{s}=60 \mathrm{~dB}$ and $\overline{\zeta(t)}=0.002$.

be able to provide phase inversion. Hence, if we assume that $k_{a}(t) \geq 0$, there is a limit on the maximum $\zeta$, which sets a maximum limit on $f_{q}$. On the other hand, the quench frequency has a lower limit because gain becomes bigger with lower quench frequencies. To compensate this, the quench amplitude has to be greatly reduced, which is not easy to control in practice. From another point of view, operating at low quench amplitudes means that we almost have a regenerative receiver, which is well-known to be not easy to control. To include this kind of restriction, in Fig. 11 we have imposed the limit $K_{s}>10$. As can be seen, these limits are not the same for different quench waveforms.

For reasonably low quench frequencies the quench signal can be generated digitally, allowing a significant amount of flexibility. However, depending on the sampling frequency of the DA converter, significant effects of quench discretization have been reported [24].

\section{Simulations}

One of the reasons why the SR principle is not well understood probably lies in the fact that it is not even easy to simulate, a matter that has been covered in several places, e.g., [25]-[27]. In fact, to get some intuitive insights into SROs it may be faster to build an oscillator and start experimenting on the effects that the quench signal has on its output.

Time-domain simulations with SPICE and its derivatives are certainly possible. The computational cost (and the accuracy of the results) can vary enormously, depending on whether a simulation is performed with the extracted layout parasitics of a CMOS design or with the plain second-order core. But in any case, the widely different time scales of the quench signal and the RF carrier make this an inefficient approach if many quench cycles have to be simulated.

Frequency-domain approaches [21] assume a steady-state solution as

$$
v_{o}(t)=\sum_{k=-N}^{N} 2\left|C_{k}\right| \cos \left(\omega_{k} t+\angle C_{k}\right),
$$

with frequencies $\omega_{k}=\omega_{i}+k \omega_{q}$.

In the linear case, the solution can be computed in closed form as [21]

$$
(\mathbf{I}-\mathbf{F D}) \mathbf{c}=\mathbf{F a},
$$

where $\mathbf{I}$ is the identity matrix, $\mathbf{c}$ is the vector containing the unknowns $C_{k}$ in (15), $\mathbf{F}$ is a diagonal matrix whose elements are the frequency response $F(s)$ of Fig. 6 evaluated at $\omega_{k}$, a represents the Fourier coefficients of the input signal $v_{i}(t)$ of frequency $\omega_{i}$, and the Toeplitz matrix $\mathbf{D}$ contains the Fourier coefficients of the periodic quench signal $k_{a}(t)$ of frequency $\omega_{q}$.

The logarithmic case can be treated in a similar way [21], but a nonlinear system of equations is obtained. In any case, once the solution is found, the most significant operation parameters, and even detailed envelope and instantaneous phase trajectories, can be computed.

In practice, it has been found that the envelope analysis tools available in commercial simulators such as [28] are able to produce meaningful results at a reduced computational cost, compared to time-domain simulations. Specific implementations of envelope-domain techniques [29], [30], taylored for the SR case, have not yet been reported. Details of envelopedomain techniques [29], [30] applied to the SR case have not yet been reported. Alternatively, the frameworks described in [11] or [18] already allow efficient modeling and simulation of the SRO's response.

\section{OSCILLATOR RERADIATION}

As the SRO is generating RF pulses of appreciable amplitude at or near the reception frequency, a SR receiver may be a source of interference for nearby receivers. The role of the LNA amplifier depicted in Fig. 6 is twofold. On the one hand, it has to perform as a true low-noise amplifier. Provided that its gain is high enough, the overall noise figure of the receiver will be given by this stage. On the other hand, the LNA should exhibit high reverse isolation. A cascode structure may offer sufficient rejection, with levels at the antenna connector of $-80 \mathrm{dBm}$ in the $\mathrm{HF}$ band, $-75 \mathrm{dBm}$ at $1 \mathrm{GHz}$, and $-63 \mathrm{dBm}$ in the $2.4 \mathrm{GHz}$ band being reported [15], [31], [32]. Additionally, the LNA may be activated only during the sensitivity period, which contributes to lowering the overall consumption. Furthermore, it is possible to insert a directional coupler in the path from LNA to SRO to provide increased reverse isolation [33].

It is also possible to minimize the in-band radiation by frequency-modulating the SRO pulse away from $\omega_{0}$ after the sensitivity period but before the amplitude has grown appreciably [34]. If this idea is implemented with a varactorbased VCO, the envelope of the response also depends on the variation of the capacitance: if the capacitance is decreased (modulation towards higher frequencies) after the sensitivity window, the envelope exhibits an even higher slope. Thus, this idea can help in achieving higher gain -provided that the active elements are able to sustain the oscillations at these higher frequencies. In [34], it was possible to modulate the SRO response more than $60 \mathrm{MHz}$ away from the receiver's $880 \mathrm{MHz}$ pass band. It is also possible to frequency-modulate 
the SRO pulses down, perhaps with the objective of simplifying further signal processing, at the expense of reduced gain.

\section{EXTERNAL QUENCH VS SELF-QUENCH}

In many implementations the quench signal is generated by a separate, external (to the SRO) quench generator. In this way, all quench parameters are fixed by design. Above, in section IV, we commented that it may be necessary to adjust the receiver's gain to keep it operating in the linear mode. This is usually done by averaging the output pulse envelope and building a feedback loop to control, for instance, the dc value of the sawtooth signal used for quenching.

One could also quench the oscillations once the SRO pulse envelope reaches a prescribed level. When operating in this mode, longer time is needed to reach the threshold for low input signal levels, resulting in a low quench frequency. In contrast, the response to high input signal levels reaches the threshold quickly, resulting in a high quench frequency. Operating in this way, the SRO performs amplitude detection by translating amplitude modulation into quench frequency modulation.

This idea can be implemented with a separate quench oscillator controlled by the SRO output, but clever circuits have been described that achieve this operation with a few components around the main SRO [2]. These are usually called self-quenched SR receivers. Of course, in these highly optimized implementations there are very few degrees of freedom left and we can not expect to achieve the same performance as with an external quench generator.

\section{PhASE AND FREQUENCY DETECTION}

As the SRO is a linear, albeit time-varying, circuit, it is clear that the response to $-x(t)$ is the negative of the response to $x(t)$ suggesting the ability to process BPSK modulations. But a detailed look at (12) further shows that the input phase term $\phi$ is directly transferred to the output pulse! It is worth pointing out that this transfer of phase information from the input to the output happens irrespective of the type of quench signal. This mechanism also explains the switched injection-locked oscillator concept [35], which was originally thought to rely on a quick switching action. It follows from (12) that this is not a requirement and, from the discussion in section IV, it may even be undesirable if high selectivity is sought.

The SRO generates pulses at $\omega_{0}$ with the phase that best matches the input signal during the observation window.

From a qualitative point of view, one can see the SRO as a device that is only able to produce a sinusoidal signal at $\omega_{0}$ and produces the particular phase that best matches the observed input signal during its sensitivity period. This property can be exploited to directly build an active backscatter transponder that produces a pulsed response that is phase-coherent with the received signal [36]. In a communication context, the SRO can be a useful front-end for detecting phase-modulated signals.

To our knowledge, this property of a SRO was never exploited until [37], probably because of the lack of a simple enough mechanism to extract phase information from the output pulses. In [37] the authors built a transmission line oscillator, where the transmission line supported two modes of oscillation depending on a control signal. In the first mode of oscillation, the generated signal has no DC component and is an exponentially growing oscillation whose phase is coherent with the phase of the received signal, as in a conventional SRO. At a given instant, the circuit topology is switched to generate the second oscillation mode, characterized by producing a waveform consisting of the sum of a) a similar waveform whose frequency is twice the frequency of the first mode and b) an exponentially growing low frequency component whose amplitude is proportional to the cosine of the signal phase in the first topology at the moment of switching. Low-pass filtering of the generated signals allows the retrieval of a DC component whose sign is used to decide the received bit in a BPSK modulation.

Instead on relying on two oscillation modes, in [38] a simple and less cumbersome method was sought: the output phase was directly sampled with a D-type flip-flop that received a single clock pulse when the output amplitude was stabilized, always at a fixed delay from the quench signal. In fact, this can be seen as a double application of the SR principle: a second-order SRO followed by a first-order regenerative comparator (the flip-flop) [39]. This idea was further refined in [40] where the SRO signal was sub-sampled with a D-type flip-flop at a frequency lower (or even substantially lower) than $\omega_{0}$ to get a shift-register filled with a set of 1-bit samples that represents the SRO output phase. The complexity of the required digital hardware is orders of magnitude simpler than with conventional IQ receivers and allows building of complete BPSK, QPSK, or 8PSK receivers [41] with very few resources, without spoiling the main point of SR reception: simplicity. The same net effect can be achieved by sampling several RCdelayed versions of the SRO output [42]-[44].

The distinct instantaneous phase trajectories associated with a frequency modulated signal can be sampled by a phasedetecting SRO [31]. In this way the SRO can efficiently detect Sunde's FSK or MSK (which can be seen as a particular case of FSK). These abilities pave the way for applications in sensor networks where SR receivers may be able to play a significant role.

An interesting feature of SROs intended for phase or frequency detection is that they do not need to rely on the operation in the linear mode, which is cumbersome to maintain, as outlined in section IV. In these applications it is possible to let the SRO operate in logarithmic mode, making its behavior insensitive to temperature and parameter tolerances and with the added benefit of higher output signal levels, typically 200 to $500 \mathrm{mV}$ (higher values are achievable although rarely necessary). The linear mode has lower consumption but its relatively low output levels ( 10 to $40 \mathrm{mV}$ ) usually require some amplifier stage further on in the processing chain, partially offsetting this advantage.

\section{NOISE}

From a block-diagram point of view, the equivalent additive output noise source of the variable-gain amplifier, $N_{S R O}$ (the 
times $k_{a}(t)$ block), in Fig. 6 can be moved to the left of the adder, so that the relevant noise sources are those of the antenna $N_{0}$, the LNA, $N_{L N A}$, and $N_{S R O}$. As usual, the latter can be neglected if the LNA provides enough gain.

Being time-varying, an exact analysis of the noise generated in the SRO is far from trivial and strongly dependent on its topology. It has been shown, however, that sufficiently accurate results can be obtained considering stationary noise sources with the values obtained at the zero crossing of $\zeta(t)$ [45].

Regarding noise, the most relevant parameter, namely the equivalent noise bandwidth, is, perhaps not surprisingly, dependent on the sensitivity function $s(t)$ and given by [46]

$$
B_{n}=\frac{\int_{t_{a}}^{t_{b}} s^{2}(\tau) d \tau}{\left[\int_{t_{a}}^{t_{b}} s(\tau) d \tau\right]^{2}}[\mathrm{~Hz}] .
$$

where, for common design parameters,

$$
B_{n}=\frac{\int_{t_{a}}^{t_{b}} s^{2}(\tau) d \tau}{\left[\int_{t_{a}}^{t_{b}} s(\tau) d \tau\right]^{2}} \approx \frac{\int_{-\infty}^{+\infty} s^{2}(\tau) d \tau}{\left[\int_{-\infty}^{+\infty} s(\tau) d \tau\right]^{2}} .
$$

Considering this approximation, the equivalent noise bandwidth is

$$
B_{n}[\mathrm{~Hz}] \approx \begin{cases}\frac{1}{2} \sqrt{\frac{\omega_{0} \beta}{\pi}} & \text { slope-controlled state } \\ \frac{\omega_{0}}{2\left(\frac{1}{\zeta_{+}}+\frac{1}{\zeta_{-}}\right)} & \text {step-controlled state. }\end{cases}
$$

The results (19) fully agree with [2], although they are obtained in a different way. Additional insights regarding noise can be found in [18] and [47].

Turning now to other effects, the gain's exponential dependence with time suggests that the quench signal jitter may have an impact on the amplitude of the output pulses. A Gaussian variation of $t_{b}$ translates into a lognormal amplitude distribution, which may be approximated again by a Gaussian distribution if the jitter is small compared to the nominal value of $t_{b}$ [4]. However, under normal conditions, which include reasonable jitter specifications, this effect has been found negligible in comparison with other noise sources [4], [45].

For phase detection, if the quench signal is also used as the symbol clock, which is the most straightforward implementation, its jitter directly translates into phase noise and has to be considered, as in any digital receiver. In the self-mixing approach of [48], the current pulse is multiplied by a symboltime-delayed version (via an analog delay) of the previous one. When this is possible (high enough symbol rate), the effects of quench jitter can be ignored because the SRO generates pulses that are phase-coherent with the input regardless of the exact quench position. In this case, degradation due to clock jitter will eventually happen later in the signal processing chain, not at the SR stage.

\section{TREnds AND AppliCATIONS}

\section{A. Oscillator Stabilization}

The stability of the reception frequency is dependent on the stability of the resonant circuit around which the transfer function $F(s)$ is built. LC circuits typically exhibit very poor stability, another reason why the SR receiver was almost abandoned. A time-shared PLL was described in [49] where the generated oscillation is compared and eventually locked to a crystal reference. As most recent implementations are actually based on a VCO for frequency tuning, this approach is the way to go for current implementations. SAW elements, which exhibit inherent stability, have been reported in [50] and a low-power BAW-stabilized SR transceiver implementation was reported in [51], with the BAW (and an additional inductor) the only non-integrated elements.

\section{B. Integrated Implementations}

The first integrated implementation was reported in [52] using a $0.8 \mu \mathrm{m}$ BiCMOS process. Later, a complete CMOS design in $0.35 \mu \mathrm{m}$ was presented in [32]. Since then, several designs have been reported in different technologies with even higher frequencies, as in [53] where a $65 \mathrm{~nm}$ implementation breaking the $100 \mathrm{GHz}$ barrier was described. The simplicity and sensitivity of the SR principle has also been used for imaging applications, with an integrated SR implementation built around a metamaterial-based resonator at $135 \mathrm{GHz}$ being presented in [54].

\section{Current Applications}

One of the fields where extremely low-power consumption is of highest importance is the field of medical implant communications. In this field, the SR principle has been used in several implementations of receivers and transceivers as, for instance, in [55]-[57]. The SR receiver has also been used as an auxiliary wake-up receiver for body area networks [58]. The idea is to use a low power consumption receiver to detect the existence of a transmission and switch to the main receiver for actual demodulation. In [59], this idea has even been used in ultrasound communications in the $\mathrm{kHz}$ range. The wireless sensor networks area may exploit the key features of SR receivers, namely reduced cost, complexity, and consumption, which are strong advantages that can outweigh its disadvantages in terms of selectivity. In this context, the $\mathrm{SR}$ receiver has recently been shown to be able to detect MSK signals with the IEEE 802.15.4 format [60] thanks to the combination of an SR receiver plus a simple synchronization loop. Thus, SR-based sensor networks may soon be seen on the market. In a related context, UWB applications have already been mentioned above and bandpass implementations have been described in [7], [9], [16], [61]-[63]. Applications to direct-sequence spread spectrum SR reception have also been reported [64]. In several of these publications, good energy efficiencies (measured in nJ/bit) are reported [63], [65]. Research has also been done on SR optical receivers [66], some even based on optomechanical oscillators [67].

\section{Conclusions}

The superregenerative principle exploits a succession of stable and unstable circuit states to achieve periodic sampling, filtering, and amplification of an input signal, linearly trading 
time (in the form of sampling period) for logarithmic gain (in $\mathrm{dB}$ ). Thus, a single active element can achieve the same gain as a chain of low-gain stages. The manner in which the system evolves from the stable to the unstable state determines the so-called sensitivity function, which, in turn, is responsible for almost all the significant parameters of the SRO: gain, bandwidth, output pulse shape, etc. An SRO with Gaussian sensitivity function can be a matched filter for a transmitted signal consisting of Gaussian pulses, meaning that there is no inherent drawback in the (synchronous) SR receiver. When compared to conventional signals, whether spread-spectrum, UWB, PSK or FSK, the SR receiver may still be a competitive choice when taking cost, simplicity, and power consumption into account, as is confirmed by renewed activity in the field.

\section{REFERENCES}

[1] E. Armstrong, "Some recent developments of regenerative circuits," Proceedings of the Institute of Radio Engineers, vol. 10, no. 4, pp. 244 260, Aug 1922.

[2] J. R. Whitehead, Super-Regenerative Receivers. Cambridge, U.K: Cambridge Univ. Press, 1950.

[3] T. H. Lee, The Design of CMOS Radio-Frequency Integrated Circuits. Cambridge, U.K: Cambridge University Press, 2004.

[4] P. Pala-Schonwalder, F. X. Moncunill-Geniz, J. Bonet-Dalmau, F. del Aguila-Lopez, and R. Giralt-Mas, "Baseband superregenerative amplification," IEEE Transactions on Circuits and Systems I: Regular Papers, vol. 56, no. 9, pp. 1930-1937, Sept 2009.

[5] R. Rieger and N. Sulistiyanto, "Integrated circuit for super-regenerative low-frequency amplification," IEEE Transactions on Circuits and Systems II: Express Briefs, vol. 65, no. 1, pp. 31-35, Jan 2018

[6] F. X. Moncunill-Geniz, J. Bonet-Dalmau, P. Pala-Schonwalder, F. del Aguila-Lopez, and R. Giralt-Mas, "A high-resolution UWB IR superregenerative receiver front end with an SRD quench shaper," IEEE Transactions on Circuits and Systems II: Express Briefs, vol. 59, no. 2, pp. 93-97, Feb 2012.

[7] M. Pelissier, D. Morche, and P. Vincent, "Super-regenerative architecture for UWB pulse detection: From theory to RF front-end design," IEEE Transactions on Circuits and Systems I: Regular Papers, vol. 56, no. 7, pp. 1500-1512, July 2009.

[8] F. X. Moncunill-Geniz, P. Pala-Schonwalder, J. Bonet-Dalmau, F. del aguila Lopez, and R. Giralt-Mas, "Sub-nanosecond pulse filtering and amplification through first-order controlled circuit instability," in 2009 European Microwave Conference (EuMC), Sept 2009, pp. 1319 1322.

[9] S. M. Fatemi, M. Sharifkhani, and A. Fotowat-Ahmady, "A unified solution for super-regenerative systems with application to correlatorbased UWB transceivers," IEEE Transactions on Circuits and Systems I: Regular Papers, vol. 62, no. 4, pp. 1033-1041, Apr 2015.

[10] F. Moncunill-Geniz, P. Pala-Schonwalder, and O. Mas-Casals, "A generic approach to the theory of superregenerative reception," Circuits and Systems I: Regular Papers, IEEE Transactions on, vol. 52, no. 1, pp. 54-70, Jan 2005.

[11] D. Frey, "Improved super-regenerative receiver theory," Circuits and Systems I: Regular Papers, IEEE Transactions on, vol. 60, no. 12, pp. 3267-3278, 2013

[12] H. P. Kalmus, "Some notes on superregeneration with particular emphasis on its possibilities for frequency modulation," Proceedings of the IRE, vol. 32, no. 10, pp. 591-600, 1944.

[13] M. Anis, M. Ortmanns, and N. Wehn, "A $2.5 \mathrm{mw} 2 \mathrm{mb} / \mathrm{s}$ fully integrated impulse-FM-UWB transceiver in $0.18 \mu \mathrm{m}$ CMOS," in 2011 IEEE MTT-S International Microwave Symposium, June 2011, pp. 1-3.

[14] J. Ayers, K. Mayaram, and T. S. Fiez, "A low power BFSK superregenerative transceiver," in 2007 IEEE International Symposium on Circuits and Systems, May 2007, pp. 3099-3102.

[15] F. Moncunill-Geniz, P. Pala-Schonwalder, C. Dehollain, N. Joehl, and M. Declercq, "An 11-Mb/s 2.1-mw synchronous superregenerative receiver at $2.4 \mathrm{GHz}$," Microwave Theory and Techniques, IEEE Transactions on, vol. 55, no. 6, pp. 1355-1362, Jun 2007.

[16] M. Anis, R. Tielert, and N. Wehn, "Super-regenerative UWB impulse detector with synchronized quenching mechanism," in ESSCIRC 2008 34th European Solid-State Circuits Conference, Sept 2008, pp. 390-393.
[17] J. H. Kim and M. M. Green, "A 0.3 nJ/bit super-regenerative pulse UWB receiver with track and detection," in 2016 14th IEEE International New Circuits and Systems Conference (NEWCAS), June 2016, pp. 1-4.

[18] J. Bohorquez, A. Chandrakasan, and J. Dawson, "Frequency-domain analysis of super-regenerative amplifiers," Microwave Theory and Techniques, IEEE Transactions on, vol. 57, no. 12, pp. 2882-2894, Dec 2009.

[19] M. Belkin and V. Bogdanenko, "Optimum filtering of pulse signals in super-regenerative receivers," Radiotekhnika, vol. 24, no. 8, pp. 97-98, Aug 1969.

[20] V. M. G.I. Kravchencko and L. Mochalina, "Investigation of a superregenerative signal detector," Telecommunications and Radio Engineering (Part 2), vol. 31, no. 5, pp. 57-60, May 1976.

[21] J. Bonet-Dalmau, F. Moncunill-Geniz, P. Pala-Schonwalder, F. del Aguila-Lopez, and R. Giralt-Mas, "Frequency domain analysis of superregenerative receivers in the linear and the logarithmic modes," Circuits and Systems I: Regular Papers, IEEE Transactions on, vol. 59, no. 5, pp. 1074-1084, May 2012.

[22] P. Pala-Schonwalder, F. X. Moncunill-Geniz, F. Aguila-Lopez, J. BonetDalmau, and R. Giralt-Mas, "A simple and robust super-regenerative oscillator for the $2.4 \mathrm{GHz}$ ISM band," in 2004 IEEE International Symposium on Circuits and Systems (IEEE Cat. No.04CH37512), vol. 4, May 2004, pp. IV-389-92 Vol.4.

[23] F. Fernandez-Rodriguez and E. Sanchez-Sinencio, "Advanced quenching techniques for super-regenerative radio receivers," Circuits and Systems I: Regular Papers, IEEE Transactions on, vol. 59, no. 7, pp. $1533-1545$, Jul 2012.

[24] P. Palà-Schönwälder, J. Bonet-Dalmau, F. del Águila López, R. Sanahuja, and F. X. Moncunill-Geniz, "Effects of quench discretization on superregenerative oscillators," in 2012 IEEE International Symposium on Circuits and Systems, May 2012, pp. 1303-1306.

[25] A. Vouilloz, C. Dehollain, and M. Declerq, "Modelisation and simulation of integrated super-regenerative receivers," in Electronics, Circuits and Systems, 1999. Proceedings of ICECS '99. The 6th IEEE International Conference on, vol. 1, 1999, pp. 521-524 vol.1.

[26] R. Feick and O. Rojas, "Modeling and simulation of the superregenerative receiver," IEEE Transactions on Consumer Electronics, vol. 43, no. 2, pp. 92-102, May 1997.

[27] T. S. Chakraborty, S. S. Yedlapalli, A. Naniyat, Y. J. Hong, and S. Kim, "On the modeling of low pass characteristics of super-regenerative receiver for high speed simulation," in 2011 IEEE Vehicular Technology Conference (VTC Fall), Sept 2011, pp. 1-6.

[28] K. Technologies. (2014) Circuit envelope element. [Online]. Available: https://literature.cdn.keysight.com/litweb/pdf/5990-3809EN.pdf

[29] E. de Cos, A. Suarez, and S. Sancho, "Envelope transient analysis of self-oscillating mixers," IEEE Transactions on Microwave Theory and Techniques, vol. 52, no. 4, pp. 1090-1100, April 2004.

[30] M. A. Farhan, E. Gad, M. S. Nakhla, and R. Achar, "High order and astable envelope following method for transient simulations of oscillatory circuits," IEEE Transactions on Microwave Theory and Techniques, vol. 62, no. 12, pp. 3309-3317, Dec 2014.

[31] P. Pala-Schonwalder, J. Bonet-Dalmau, A. Lopez-Riera, F. MoncunillGeniz, F. del Aguila-Lopez, and R. Giralt-Mas, "Superregenerative reception of narrowband FSK modulations," Circuits and Systems I: Regular Papers, IEEE Transactions on, vol. 62, no. 3, pp. 791-798, Mar 2015.

[32] A. Vouilloz, M. Declercq, and C. Dehollain, "A low-power CMOS superregenerative receiver at $1 \mathrm{GHz}$," IEEE Journal of Solid-State Circuits, vol. 36, no. 3, pp. 440-451, Mar 2001.

[33] R. Dickerson, J. Chess, and J. Lorthioir, "Superregenerative circuit," Jul. 16 2002, US Patent 6,421,535. [Online]. Available: https://www.google.es/patents/US6421535

[34] P. Palà-Schönwälder, J. Bonet-Dalmau, F. X. Moncunill-Geniz, F. del Águila López, and R. Giralt-Mas, "A low in-band radiation superregenerative oscillator," IEEE Transactions on Circuits and Systems II: Express Briefs, vol. 60, no. 6, pp. 307-310, June 2013.

[35] M. Vossiek and P. Gulden, "The switched injection-locked oscillator: A novel versatile concept for wireless transponder and localization systems," IEEE Transactions on Microwave Theory and Techniques, vol. 56, no. 4, pp. 859-866, April 2008.

[36] A. Strobel, C. Carlowitz, R. Wolf, F. Ellinger, and M. Vossiek, "A millimeter-wave low-power active backscatter tag for FMCW radar systems," IEEE Transactions on Microwave Theory and Techniques, vol. 61, no. 5, pp. 1964-1972, May 2013

[37] L. Hernandez and S. Paton, "A superregenerative receiver for phase and frequency modulated carriers," in Circuits and Systems, 2002. ISCAS 
2002. IEEE International Symposium on, vol. 3, 2002, pp. III-81-III84 vol.3.

[38] P. Pala-Schonwalder, F. Moncunill-Geniz, J. Bonet-Dalmau, F. del Aguila-Lopez, and R. Giralt-Mas, "A BPSK superregenerative receiver. preliminary results," in Circuits and Systems, 2009. ISCAS 2009. IEEE International Symposium on, May 2009, pp. 1537-1540.

[39] A. Abidi and H. Xu, "Understanding the regenerative comparator circuit," in Proceedings of the IEEE 2014 Custom Integrated Circuits Conference, Sept 2014, pp. 1-8.

[40] P. Pala-Schonwalder, J. Bonet-Dalmau, F. Xavier Moncunill-Geniz, F. del Aguila-Lopez, and R. Giralt-Mas, "A superregenerative QPSK receiver," Circuits and Systems I: Regular Papers, IEEE Transactions on, vol. 61, no. 1, pp. 258-265, Jan 2014.

[41] J. Bonet-Dalmau, A. Lopez-Riera, P. Palà-Schonwalder, F. X. MoncunillGeniz, and A. Babi-Oller, "Design and performance comparison of a superregenerative MPSK transceiver," in 2015 IEEE International Conference on Electronics, Circuits, and Systems (ICECS), Dec 2015, pp. 169-172.

[42] G. Ibrahim, A. Hafez, A. Khalil, and H. Elsimary, "A 2.7 GHz superregenerative receiver front-end for QPSK modulated signals," in Communication Systems, Networks Digital Signal Processing (CSNDSP), 2012 8th International Symposium on, Jul 2012, pp. 1-4.

[43] G. H. Ibrahim and A. N. Hafez, "An 8-PSK digital phase detection technique for super-regenerative receivers," in 2015 IEEE International Conference on Electronics, Circuits, and Systems (ICECS), Dec 2015, pp. 240-243.

[44] R. Mirzalou and M. F. Wagdy, "An 8-PSK super regenerative receiver with new phase detection technique," in 2016 12th International Computer Engineering Conference (ICENCO), Dec 2016, pp. 14-19.

[45] D. G. Lee and P. P. Mercier, "Noise analysis of phase-demodulating receivers employing super-regenerative amplification," IEEE Transactions on Microwave Theory and Techniques, vol. 65, no. 9, pp. 3299-3311, Sept 2017.

[46] A. López-Riera, "Aplicaciones avanzadas del principio superregenerativo a comunicaciones por radiofrecuencia," Ph.D. dissertation, Universitat Politècnica de Catalunya, Manresa, 2017.

[47] P. Thoppay, C. Dehollaini, and M. Declercq, "Noise analysis in superregenerative receiver systems," in Research in Microelectronics and Electronics, 2008. PRIME 2008. Ph.D., Jun 2008, pp. 189-192.

[48] C. Carlowitz, A. Esswein, R. Weigel, and M. Vossiek, "Regenerative sampling self-mixing receiver: A novel concept for low complexity phase demodulation," in 2013 IEEE MTT-S International Microwave Symposium Digest (MTT), June 2013, pp. 1-4.

[49] N. Joehl, C. Dehollain, P. Favre, P. Deval, and M. Declerq, "A low-power 1-GHz super-regenerative transceiver with time-shared PLL control," IEEE Journal of Solid-State Circuits, vol. 36, no. 7, pp. 1025-1031, Jul 2001.

[50] D. L. Ash, "A low cost superregenerative SAW stabilized receiver," IEEE Transactions on Consumer Electronics, vol. CE-33, no. 3, pp. 395-404, Aug 1987.

[51] B. Otis, Y. H. Chee, and J. Rabaey, "A $400 \mu \mathrm{W}-\mathrm{RX}$, 1.6mw-TX superregenerative transceiver for wireless sensor networks," in ISSCC. 2005 IEEE International Digest of Technical Papers. Solid-State Circuits Conference, 2005., Feb 2005, pp. 396-606 Vol. 1.

[52] P. Favre, N. Joehl, A. Vouilloz, P. Deval, C. Dehollaini, and M. Declercq, "A 2-V 600- $\mu$ a 1-GHz BiCMOS super-regenerative receiver for ISM applications," Solid-State Circuits, IEEE Journal of, vol. 33, no. 12, pp. 2186-2196, Dec 1998.

[53] G. Feng, C. C. Boon, F. Meng, and X. Yi, "A 100-GHz 0.21-K NETD $0.9-\mathrm{mW} /$ pixel charge-accumulation super-regenerative receiver in $65-\mathrm{nm}$ CMOS," IEEE Microwave and Wireless Components Letters, vol. 26, no. 7, pp. 531-533, Jul 2016.

[54] Y. Shang, H. Yu, S. Hu, Y. Liang, X. Bi, and M. A. Arasu, "Highsensitivity CMOS super-regenerative receiver with quench-controlled high-Q metamaterial resonator for millimeter-wave imaging at 96 and $135 \mathrm{GHz}, "$ IEEE Transactions on Microwave Theory and Techniques, vol. 62, no. 12, pp. 3095-3106, Dec 2014.

[55] C. Ma, C. Hu, J. Cheng, L. Xia, and P. Y. Chiang, "A near-threshold, 0.16 $\mathrm{nJ} / \mathrm{b}$ OOK-transmitter with $0.18 \mathrm{~nJ} / \mathrm{b}$ noise-cancelling super-regenerative receiver for the medical implant communications service," IEEE Transactions on Biomedical Circuits and Systems, vol. 7, no. 6, pp. 841-850, Dec 2013

[56] J. Bohorquez, A. Chandrakasan, and J. Dawson, "A $350 \mu \mathrm{W}$ CMOS MSK transmitter and $400 \mu \mathrm{W}$ OOK super-regenerative receiver for medical implant communications," Solid-State Circuits, IEEE Journal of, vol. 44, no. 4, pp. 1248-1259, Apr 2009.
[57] T. Copani, S. Min, S. Shashidharan, S. Chakraborty, M. Stevens, S. Kiaei, and B. Bakkaloglu, "A CMOS low-power transceiver with reconfigurable antenna interface for medical implant applications," IEEE Transactions on Microwave Theory and Techniques, vol. 59, no. 5, pp. 1369-1378, May 2011.

[58] J. Petäjäjärvi, H. Karvonen, R. Vuohtoniemi, M. Hämäläinen, and M. Huttunen, "Preliminary study of superregenerative wake-up receiver for WBANs," in 2014 8th International Symposium on Medical Information and Communication Technology (ISMICT), April 2014, pp. 1-5.

[59] H. Fuketa, S. O'uchi, and T. Matsukawa, "A $0.3-\mathrm{V} \quad 1-\mu \mathrm{W}$ superregenerative ultrasound wake-up receiver with power scalability," IEEE Transactions on Circuits and Systems II: Express Briefs, vol. 64, no. 9, pp. 1027-1031, Sept 2017.

[60] A. López-Riera, F. del Águila López, P. Palá-Schönwälder, J. BonetDalmau, R. Giralt-Mas, and F. X. Moncunill-Geniz, "Joint symbol and chip synchronization for a burst-mode-communication superregenerative MSK receiver,' IEEE Transactions on Circuits and Systems I: Regular Papers, vol. 64, no. 5, pp. 1260-1269, May 2017.

[61] D. G. Lee and P. P. Mercier, "A $1.65 \mathrm{~mW}$ PLL-free PSK receiver employing super-regenerative phase sampling," in Biomedical Circuits and Systems Conference (BioCAS), 2015 IEEE, Oct 2015, pp. 1-4.

[62] F. X. Moncunill, P. Palà-Schönwälder, J. Bonet-Dalmau, F. Aguila, and M. Giralt, "Ultra wideband impulse radio superregenerative reception," in Ultra wideband communications : novel trends - system, architecture and implementation, M. Matin, Ed. Intech, 2011, pp. 113-136.

[63] P. Thoppay, C. Dehollain, M. Green, and M. Declercq, "A 0.24-nJ/bit super-regenerative pulsed UWB receiver in $0.18-\mu \mathrm{m}$ CMOS," SolidState Circuits, IEEE Journal of, vol. 46, no. 11, pp. 2623 -2634, Nov 2011.

[64] F. X. Moncunill-Geniz and P. Pala-Schonwalder, "A DSSS superregenerative receiver with tau-dither loop," in Wireless Technology, 2004. 7th European Conference on, Oct 2004, pp. 349-352.

[65] Y. Zheng, Y. Zhu, C. W. Ang, Y. Gao, and C. H. Heng, "A 3.54 nJ/bitRX, $0.671 \mathrm{~nJ} / \mathrm{bit}-\mathrm{TX}$ burst mode super-regenerative UWB transceiver in $0.18-\mu \mathrm{m}$ CMOS," IEEE Transactions on Circuits and Systems I: Regular Papers, vol. 61, no. 8, pp. 2473-2481, Aug 2014.

[66] M. C. España-Boquera and A. Puerta-Notario, "Bit-error rate and frequency response in superregenerative semiconductor laser receivers," Opt. Lett., vol. 24, no. 3, pp. 157-159, Feb 1999. [Online]. Available: http://ol.osa.org/abstract.cfm?URI=ol-24-3-157

[67] T. Beyazoglu, T. O. Rocheleau, A. J. Grine, K. E. Grutter, M. C. Wu, and C. T. C. Nguyen, "A super-regenerative optical receiver based on an optomechanical oscillator," in 2015 28th IEEE International Conference on Micro Electro Mechanical Systems (MEMS), Jan 2015, pp. 976-979. 